\title{
Analysis of Combined Effect of CYP2C19 Genetic Polymorphism and Proton Pump Inhibitors Coadministration on Trough Concentration of Voriconazole
}

\author{
Magesa Mafuru (D) ${ }^{1,2}$ \\ Sanlan $\mathrm{Wu}^{2}$ \\ Henry Mayala ${ }^{2}$ \\ Zaituni Msengwa ${ }^{3}$ \\ Amani Phillip' \\ Charles Mgone'
}

'Department of Clinical Pharmacology \& Therapeutics, Hubert Kairuki Memorial University, Dar es Salaam, Tanzania; ${ }^{2}$ Department of Pharmacy, Union Hospital of Huazhong University of Science and Technology, Wuhan, People's Republic of China; ${ }^{3}$ Department of Biological and Preclinical Studies, Muhimbili University of Health and Allied Sciences, Dar es Salaam, Tanzania
Correspondence: Magesa Mafuru Department of Clinical Pharmacology \& Therapeutics, Faculty of Medicine, Hubert Kairuki Memorial University, P.O.Box 65300 , Dar es Salaam, Tanzania Tel +2557439I7I72

Email mmafuru@gmail.com; magesa. mafuru@hkmu.ac.tz
Purpose: To analyze the combined effect of CYP2C19 genetic polymorphism and PPIs coadministration on voriconazole trough concentration $\left(\mathrm{VCZ}-\mathrm{C}_{\text {trough }}\right)$ in Chinese patients with hematological disorders.

Patients and Methods: A prospective observational study involved 250 plasma samples from 114 adult patients receiving voriconazole with or without PPIs were analyzed. Demographics and clinical characteristics were obtained from patient's records. A validated LC-MS/MS was used to quantify the plasma $\mathrm{VCZ}-\mathrm{C}_{\text {trough }}$. Genotyping for CYP2C19*2 and CYP2C19*3 variant alleles was performed by PCR-RFLP followed by DNA sequencing. The combined total score (from 2 to 5) was calculated for each patient. The higher the score, the lesser the metabolism of the patient.

Findings: Fifty percent of patients administered with voriconazole were coadministered with PPIs, predominantly omeprazole or esomeprazole. Patients exhibiting CYP2C19 poor metabolizer phenotype showed a significantly higher median $\mathrm{VCZ}_{\text {trough }}$, $(4.31 \mu \mathrm{g} / \mathrm{mL} \quad[\mathrm{IQR}, 1.64 \mu \mathrm{g} / \mathrm{mL}-7.36 \mu \mathrm{g} / \mathrm{mL}])$ than patients with normal metabolizer $(1.38 \mu \mathrm{g} / \mathrm{mL}, \quad[\mathrm{IQR}, \quad 0.79 \mu \mathrm{g} / \mathrm{mL}-2.14 \mu \mathrm{g} / \mathrm{mL}], \quad \mathrm{p}<0.0001)$. Similarly, patients coadministration with PPIs had higher median VCZ-C trough $(2.86 \mu \mathrm{g} / \mathrm{mL}[\mathrm{IQR} 1.33 \mu \mathrm{g} / \mathrm{mL}-$ $4.66 \mu \mathrm{g} / \mathrm{mL}])$, than PPIs non-users $(1.71 \mu \mathrm{g} / \mathrm{mL}$, [IQR, $0.86 \mu \mathrm{g} / \mathrm{mL}-3.48 \mu \mathrm{g} / \mathrm{mL}], \mathrm{p}=$ 0.001). However, we noted that the median $\mathrm{VCZ}-\mathrm{C}_{\text {trough }}$ for each factor was ranging within the normal recommended therapeutic range in the Chinese population $(0.5 \mu \mathrm{g} / \mathrm{mL}-$ $5 \mu \mathrm{g} / \mathrm{mL}$ ). But when the two factors were combined, the median VCZ- $\mathrm{C}_{\text {trough }}$ was steadily increasing as the metabolic capacity (reflected by combined total score) was increasing. Importantly, the median $\mathrm{VCZ}-\mathrm{C}_{\text {trough }}$ in $\mathrm{PM} / \mathrm{PPIs}$ user (total score 5) was significantly elevated to supra-therapeutic levels compared to NM/PPI non-user group (total score 2) $(5.83 \mu \mathrm{g} / \mathrm{mL}$ [IQR, $2.19 \mu \mathrm{g} / \mathrm{mL}-9.51 \mu \mathrm{g} / \mathrm{mL}$ ] versus $1.13 \mu \mathrm{g} / \mathrm{mL}$ [IQR, $0.67 \mu \mathrm{g} / \mathrm{mL}-1.82 \mu \mathrm{g} /$ $\mathrm{mL}]$ ), respectively, $\mathrm{P}<0.0001$. Furthermore, we observed that the elevation of median VCZ-C $\mathrm{C}_{\text {trough }}$ to supra-therapeutic levels was largely contributed by omeprazole or esomeprazole compared to lansoprazole or pantoprazole.

Conclusion: Coadministration with PPIs significantly increased voriconazole trough concentrations and there was an additive effect in CYP2C19 PMs, who were most likely to have supra-therapeutic levels.

Keywords: voriconazole, proton pump inhibitors, CYP2C19 polymorphism, drug-drug interaction, drug-disease interaction 


\section{Introduction}

Voriconazole is a second-generation triazole antifungal agent with broad-spectrum activities against numerous fungal infections ${ }^{1,2}$ Voriconazole has been regarded as the first-line drug for treatment and prophylaxis of invasive fungal infection (IFI) particularly invasive aspergillosis, a serious fungal infection common in patients with the declined immune system such as patients with hematologic disorders, solid organ transplantation (SOT) and hematopoietic stem cell transplantation (HSCT) patients.

Even though voriconazole has potential clinical use, its plasma concentration among individuals is highly variable. ${ }^{3}$ Since voriconazole exhibits a narrow therapeutic index, high variability of plasma concentration can adversely affect therapeutic outcomes. A set of factors including patient's age, gender, liver function status, CYP2C19 and CYP3A4 polymorphism, inflammation, and drug co-administration have been reported to influence voriconazole concentration variability. ${ }^{4,5}$

In vitro studies have shown that voriconazole is predominantly metabolized by $\mathrm{CYP} 2 \mathrm{C} 19$ and to less extent CYP3A4 to voriconazole N-oxide, (the major metabolite accounting for about $74 \%$ of all metabolites) and 4-hydroxyvoriconazole (as minor metabolites). ${ }^{6}$ It has been noted that CYP2C19 and CYP3A4 are highly polymorphic enzymes and the variant alleles with reduced enzyme function (CYP2C19*2 and CYP2C19*3) are highly distributed in Asian (12-23\%) than in Caucasians (1-6\%) and African Americans $(1-7.5 \%)^{7}$ while CYP2C19*17 which confer increased enzyme activity is highly distributed in Caucasian and African Americans (18-27\%) than in Asians (<4\%).

Proton pump inhibitors (PPIs) such as omeprazole, lansoprazole, esomeprazole, rabeprazole, and pantoprazole are commonly used medications in the treatment of acidrelated gastrointestinal disorders including gastroesophageal reflux disease, nonsteroidal anti-inflammatory druginduced gastrointestinal lesions, Zollinger-Ellison syndrome, dyspepsia, and Helicobacter pylori (in combination with antibiotics). ${ }^{8}$ PPIs are well-known substrates and inhibitors of CYP2C19; the major enzyme responsible for voriconazole metabolism. ${ }^{9-11}$ Since the acid-related disorders are high in hospitalized patients' particularly hematological-disordered patients, the likelihood of PPIs and voriconazole coadministration is possibly higher, subjecting the two drugs for drug-drug interaction. More evidence suggests that PPIs also have a role in sensitization of tumors to chemotherapy by regulating the acidic $\mathrm{pH}$ gradient of tumor-microenvironment via inhibition of the vacuolar-type $\mathrm{H}^{+}$-ATPase (V-ATPase) overexpressed in tumor cells. ${ }^{12-15}$ This effect might be one of the reasons for the increased use of PPIs in hematological tumor patients who are likely to use voriconazole as well.

In vitro and in vivo studies have reported a significant increase in voriconazole exposure upon coadministration with PPIs, ${ }^{10,16,17}$ and the extent of CYP2C19 inhibition differs among individual PPIs. ${ }^{8,11}$ Even though the influence of PPIs on the pharmacokinetic profile of voriconazole has been adequately demonstrated, little is known regarding the combined effect of CYP2C19 genetic polymorphism and PPIs on voriconazole exposure. The CYP2C19 polymorphic contribute largely to the plasma concentration variability of voriconazole. In the current study, we postulated that individual patients exhibiting CYP2C19 poor metabolizer variant allele (CYP2C19 *2/ $* 2$, $* 2 / * 3$ and $* 3 / * 3$ ) who receive PPIs have highest increased voriconazole exposure than patients exhibiting CYP2C19 normal metabolizer who receive or do not receive PPIs. This study aimed to investigate the combined effects of CYP2C19 polymorphism and PPIs use on voriconazole exposure; measured by voriconazole trough concentration ( $\mathrm{VCZ}-\mathrm{C}_{\text {trough }}$ ).

\section{Materials and Methods}

\section{Patient's Characteristics and Study Design}

One hundred and fourteen (114) adult patients admitted at Union Hospital of Tongji Medical College diagnosed with hematologic disorders; receiving voriconazole with or without PPIs participated in this study after provided with written informed consent. The clinical protocol and informed consent were approved by the local ethics committee of Tongji Medical College of Huazhong University of Sciences and Technology. A prospective observational study was conducted from June 2017 to May 2019, in accordance to the Declaration of Helsinki. The adult patients aged $\geq 18$ years old, treated with voriconazole for possible, probable, or proven IFI were consecutively included in the study. Patients with severe renal failure or concurrently administered with strong inducers or inhibitors (except PPIs) of CYP-450 isoenzymes ${ }^{18}$ were excluded from the study. Data on demographics and patient's clinical characteristics (Table 1) including data on PPIs uses were prospectively extracted from patient's medical records. 
Table I Patient's Demographics and Clinical Characteristics

\begin{tabular}{|c|c|}
\hline Characteristics & Value \\
\hline Age (yrs) & $36(25-5 I)$ \\
\hline \multicolumn{2}{|l|}{ Sex, n (\%) } \\
\hline Males & $83(73)$ \\
\hline Females & $31(27)$ \\
\hline \multicolumn{2}{|l|}{ Diagnosis, n (\%) } \\
\hline Chronic myeloid leukemia & $6(5)$ \\
\hline Acute lymphoblastic leukemia & $25(22)$ \\
\hline Acute myeloid leukemia & $26(23)$ \\
\hline Myelodysplastic syndrome & $8(7)$ \\
\hline Severe aplastic anemia & $13(11)$ \\
\hline Others & $36(32)$ \\
\hline Voriconazole trough concentration $(\mu \mathrm{g} / \mathrm{mL})$ & $2.1(1.13-4.13)$ \\
\hline \multicolumn{2}{|l|}{ Route of administration, $\mathrm{n}(\%)$} \\
\hline Oral & $202(80.8)$ \\
\hline Intravenous & $48(19.2)$ \\
\hline \multicolumn{2}{|l|}{ Drug co-administration, $\mathrm{n}(\%)$} \\
\hline Calcineurin inhibitors & $114(100)$ \\
\hline Proton pump inhibitors & $62(54)$ \\
\hline Glucocorticoids & $39(34)$ \\
\hline Others & $34(28)$ \\
\hline Total bilirubin $(\mu \mathrm{mol} / \mathrm{L})^{\dagger}$ & $10.5 \pm 5.5$ \\
\hline Albumin $(g / L)^{\dagger}$ & $38.5 \pm 5.6$ \\
\hline Globulin $(\mathrm{g} / \mathrm{L})^{\dagger}$ & $21.6 \pm 4.7$ \\
\hline Total bile acid $(\mu \mathrm{mol} / \mathrm{L})$ & $7.0(4.03-12.03)$ \\
\hline Alanine aminotransferase $(\mathrm{u} / \mathrm{L})$ & $15.0(9.0-25.75)$ \\
\hline Aspartate aminotransferase (u/L) & $18.0(13-25)$ \\
\hline Alkaline phosphatase (u/L) & $100(77-140.75)$ \\
\hline Gamma glutamyltransferase (u/L) & $62(31.25-109.0)$ \\
\hline C-reactive protein (mg/L) & $5.9(3.17-20.7)$ \\
\hline Lymphocytes (\%) & $16.8(9.9-35.4)$ \\
\hline Neutrophils (\%) & $70.7(5|.3-8| .8)$ \\
\hline Platelets (g/L) & $126(53.7-189.3)$ \\
\hline
\end{tabular}

Notes: The data are expressed as median (interquartile range) unless otherwise stated; ${ }^{\dagger}$ These data are expressed as mean \pm standard deviation.

\section{Sample Collection}

Blood samples at steady-state for measurement of voriconazole trough concentration, and CYP2C19 genotyping were aseptically collected in EDTA-containing tubes. A steady-state trough blood sample was defined as a sample collected at interval windows of $12 \mathrm{hrs}$. postdose, after three days of voriconazole treatment with a loading dose or a sample drawn after five days or more, following treatment initiation at the same interval windows for patients who did not receive loading dose. ${ }^{19,28}$ The samples were collected immediately before administration of the next voriconazole dose. The samples were centrifuged at $1500 \mathrm{~g}$ for $15 \mathrm{~min}$ to obtain plasma and blood cells. The plasma and blood cells were divided into aliquots and stored at $-80^{\circ} \mathrm{C}$ until analysis.

\section{Liquid Chromatography-Tandem Mass Spectrometry (LC-MS/MS) Detection of Voriconazole Trough Concentration}

Voriconazole (purity $\geq 99 \%$ ) was purchased from Wuhan Yuanda Pharmaceutical Group Co., Ltd (China); Propranolol (PPN) as internal standard (purity $\geq 99 \%$ ) was purchased from Aladdin Reagent Co., Ltd, (Shanghai, China); Methyl tert-butyl ether (MTBE), Chloroform (TCM), Ethyl acetate (ACE), Ammonium formate, and Dichloromethane (DCM) were obtained from Sinopharm Group Chemical Reagent Co., Ltd (China); Formic acid was purchased from Tianjin BASF Chemical Co., Ltd (China); Methanol and acetonitrile (HPLC grade) were obtained from Fisher Scientific (Fair Lawn, NJ, USA).

The aliquot portions of frozen plasma samples were used for quantification of voriconazole trough concentrations. The detection was conducted using a validated liquid chromatography-tandem mass spectrometry (LCMS/MS) method according to the previous study. ${ }^{20}$ Precision ranged from 4.1 to $7.7 \%$. The lower limit of detection was $0.072 \mu \mathrm{g} / \mathrm{mL}$. The calibration range from 0.072 to $8 \mu \mathrm{g} / \mathrm{mL}$, with correlation coefficients (r) $>$ 0.999 . The accuracy of VCZ in plasma was within $100 \%$ $\pm 12 \%$.

\section{CYP2C19 Genotyping}

Genotyping for single nucleotide polymorphism (SNP) of CYP2C19 $* 2$ and CYP2C19 *3 variant alleles was performed by polymerase chain reaction-restriction fragment length polymorphism (PCR-RFLP) and finalized by DNA sequencing. ${ }^{28}$ Genomic DNA was isolated from white blood cells using a magnetic bead genomic DNA extraction kit (Tiangen Biochemical, China) according to the manufacturer's instructions. DNA amplification by polymerase chain reaction (Applied Biosystem, Foster City, California) was conducted using primer pairs shown in Supplementary Table S1. DNA fragment sequencing was performed using the ABI Sequencer 3730XL (Applied Biosystems) by employing the Sanger sequencing method. All variant alleles were in Hardy-Weinberg equilibrium. CYP2C19 phenotype was assigned according to 
nomenclature by the Clinical Pharmacogenetics Implementation Consortium (CPIC). ${ }^{21}$ CYP2C19 *1 (wild-type) was inferred in the absence of CYP2C19*2 or CYP2C19*3 alleles. The wild-type normal metabolizer (NM) phenotype (ie, $* 1 / * 1$ ) was assigned by default to patients without $* 2$, or $* 3$, allele. Patients with one copy of $* 2$ or $* 3$ allele were classified as the intermediate metabolizer (IM), and two copies (eg, $* 2 / * 2, * 3 / * 3$ or $* 2 / * 3$ ) as poor metabolizer (PM) phenotype. Because of its low distribution in the Chinese population, CYP2C19 $* 17$ allele was not genotyped.

\section{Determination of Total Combined Score}

To determine the combined effects of both CYP2C19 polymorphism and PPIs use, a combined score was calculated. ${ }^{3}$ First, a specific score was separately assigned to each CYP2C19 allele and PPIs use status as follows: for CYP2C19 allele, patients exhibiting NM (ie, CYP2C19 $* 1 / * 1$ ) were assigned a score of 1 ; patients exhibiting IM (ie, CYP2C19 $* 1 / * 2$ or CYP2C19 $* 1 / * 3$ ) were assigned a score of 2 and patients exhibiting PM (ie, CYP2C19*2/ $* 2, * 2 / * 3$ and $* 3 / * 3$ ) were assigned a score of 3 . For PPIs use status, patients who did not use PPIs were assigned a score of 1 and those who used PPIs were assigned a score of 2. Finally, both specific scores were then added to obtain a combined total score. The total score ranged from 2 to 5 . The combined score was expressed in arbitrary units. A total of four combination groups were generated. A combined score equal to 2 corresponds to NM/PPIs non-users group; a score of 3 corresponds to NM/PPIs users or IM/PPIs non-users group; a score of 4 corresponds to IM/PPIs users or PM/PPIs non-users group, and a score of 5 corresponds to PM/PPIs users group. The higher the score, the lesser the metabolic capacity of the patient.

\section{Statistical Analysis}

Shapiro-Wilk test was used to test the normality of the data. Numerical variables are expressed as mean \pm standard deviation for normally distributed data or as the median and interquartile range (IQR) for skewed data, while categorical variables are expressed as a percentage. Comparison between two groups was performed using Mann-Whitney $U$-test for scale variables and Chi-square $\left(\chi^{2}\right)$ or Fisher's exact test for categorical data. Comparison between more than two groups was performed by Kruskal-Wallis test for scale variables. The backward multivariate linear regression model was used to determine more factors associated with voriconazole trough concentration. Data were analyzed using SPSS software, version 23. A two-tailed P-value of $<0.05$ level was considered statistically significant.

\section{Results}

\section{Demographics and Clinical Characteristics of Patients}

A total of 250 blood samples from 114 patients (average of 2 samples per patient) with a median age of 36 (IQR, 25 to 51) years were studied. Patients were diagnosed with various hematological disorders such as chronic myeloid leukemia, acute lymphoblastic leukemia, acute myeloid leukemia, myelodysplastic syndrome, severe aplastic anemia, and other diseases including non-Hodgkin lymphoma, and cardiovascular diseases co-morbidity. All patients were administered voriconazole $200 \mathrm{mg}, 12 \mathrm{hrly}$ with or without a pre-loading dose. Voriconazole was administered for either prophylaxis or treatment of invasive fungal infection and the median trough concentration was $2.1 \mu \mathrm{g} / \mathrm{mL}$ (IQR, $1.13 \mu \mathrm{g} / \mathrm{mL}$ to $4.13 \mu \mathrm{g} / \mathrm{mL}$ ). Fifty percent $(50 \%)$ of patients administered with voriconazole were coadministered with PPIs; of these 39\% were receiving omeprazole; $24 \%$ lansoprazole; $8 \%$ pantoprazole and 29\%, esomeprazole. Immunosuppressant drugs particularly calcineurin inhibitors (cyclosporine or tacrolimus) was also administered to all patients. CYP2C19 genotype comprised 39 (34.2\%) normal metabolizers, 52 (45.6\%) intermediate metabolizers and $23(20.2 \%)$ poor metabolizers. Demographics and clinical characteristics are summarized in Table 1.

\section{CYP2CI9 Gene Polymorphism/PPIs Combination Effect on Trough Concentration of Voriconazole}

The analysis of the effect of CYP2C19 gene polymorphism (as individual factor) on trough concentration of voriconazole showed a significantly higher median trough concentration $(4.31 \mu \mathrm{g} / \mathrm{mL}$ [IQR, $1.64 \mu \mathrm{g} / \mathrm{mL}$ to $7.36 \mu \mathrm{g} / \mathrm{mL}], \mathrm{n}=48)$ in patients with $\mathrm{PM}$ than in patient with NM phenotype $(1.38 \mu \mathrm{g} / \mathrm{mL},[\mathrm{IQR}, 0.79 \mu \mathrm{g} / \mathrm{mL}$ to $2.14 \mu \mathrm{g} / \mathrm{mL}], \mathrm{n}=103)$, $\mathrm{p}<0.0001$. Similarly, IM patients presented with significantly higher median trough concentration than NM patients, ie, $(3.04 \mu \mathrm{g} / \mathrm{mL},[\mathrm{IQR}, 1.66 \mu \mathrm{g} / \mathrm{mL}$ to $4.20 \mu \mathrm{g} / \mathrm{mL}$ ], $\mathrm{n}=99$ versus $1.38 \mu \mathrm{g} / \mathrm{mL}$, [IQR, $0.79 \mu \mathrm{g} / \mathrm{mL}$ to $2.14 \mu \mathrm{g} / \mathrm{mL}]$ ), respectively, $\mathrm{p}<0.0001$. Even though the PM patients had significantly higher median trough concentration than NM 
or IM counterparts, the median trough concentration of all groups was within the normal recommended therapeutic range in the Chinese population, which is $0.5 \mu \mathrm{g} / \mathrm{mL}$ to $5 \mu \mathrm{g} / \mathrm{mL}^{22,23}$ (Figure 1A). Similarly, PPIs co-administration (analyzed as an individual factor) also impacted the trough concentration of voriconazole. A significantly higher median trough concentration $(2.86 \mu \mathrm{g} / \mathrm{mL} \quad[\mathrm{IQR}, 1.33 \mu \mathrm{g} / \mathrm{mL}$ to $4.66 \mu \mathrm{g} / \mathrm{mL}], \mathrm{n}=126$ ) in PPIs users than in non-user $(1.71 \mu \mathrm{g} / \mathrm{mL},[\mathrm{IQR}, 0.86 \mu \mathrm{g} / \mathrm{mL}$ to $3.48 \mu \mathrm{g} / \mathrm{mL}], \mathrm{n}=124)$, $\mathrm{p}=0.001$ was observed. Even though the PPIs user had higher median trough concentration than the non-users, the median concentration of the two groups was as well within the recommended normal therapeutic (Figure 1B).

The combined effect of CYP2C19 gene polymorphism and PPIs co-medication on trough concentration of voriconazole was analyzed. Voriconazole trough concentration was steadily increasing as the combined total score was increasing. The median (IQR) trough concentration of $1.13 \mu \mathrm{g} / \mathrm{mL}$ $(0.67 \mu \mathrm{g} / \mathrm{mL}$ to $1.82 \mu \mathrm{g} / \mathrm{mL}), 1.96 \mu \mathrm{g} / \mathrm{mL} \quad(1.18 \mu \mathrm{g} / \mathrm{mL}$ to $3.72 \mu \mathrm{g} / \mathrm{mL}), 2.96 \mu \mathrm{g} / \mathrm{mL}(1.70$ to 4.47$)$ and $5.83 \mu \mathrm{g} / \mathrm{mL}$
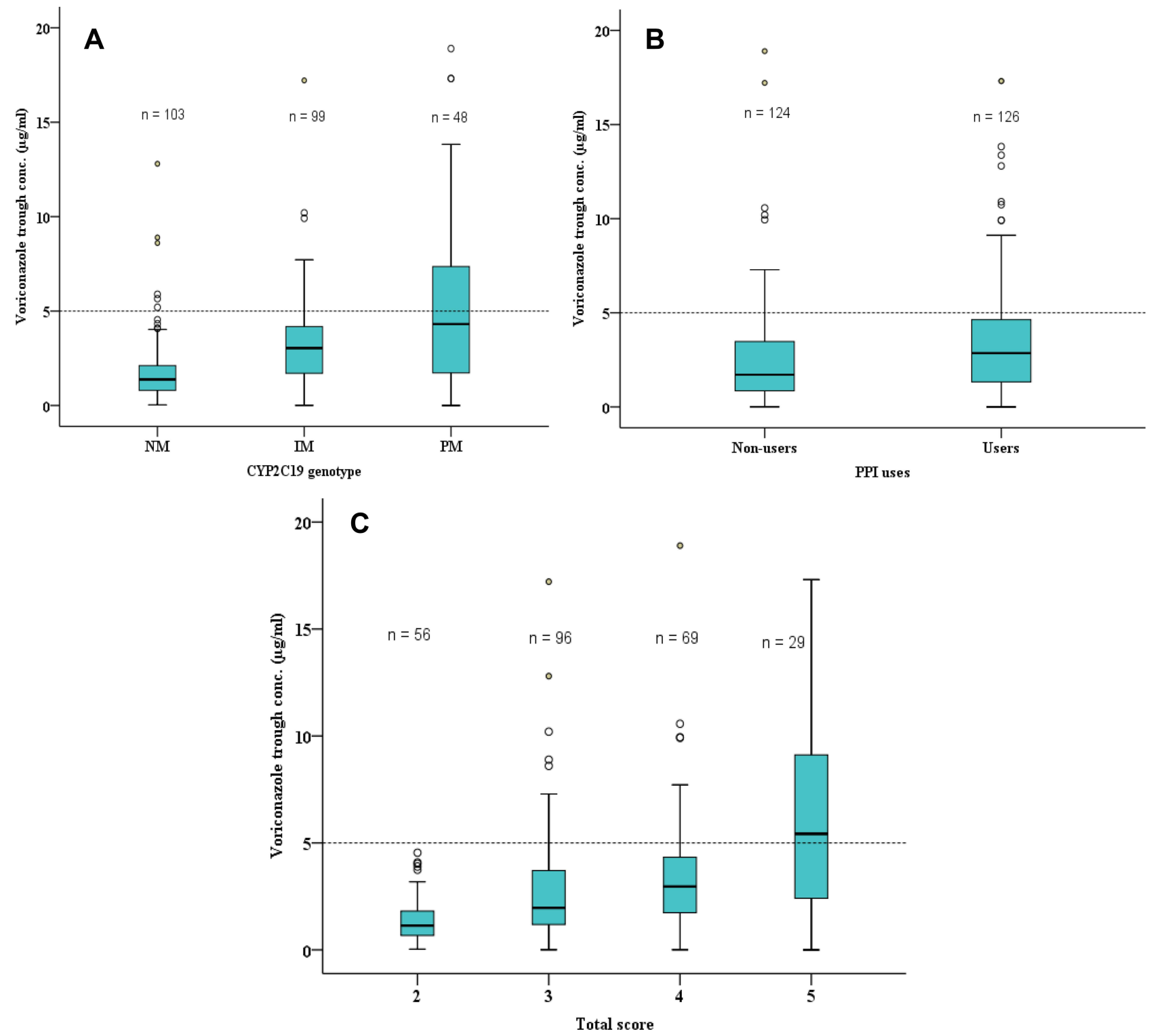

Figure I Box-and-whisker plot showing the effect of CYP2CI9 genotype and proton pump inhibitors (PPIs) use on trough concentration of voriconazole. Box (25th, median, and 75th percentiles) and whisker (5th and 95th percentiles); the small filled and unfilled circle shows outlier cases. Individual effect of CYP2CI 9 genotype on trough concentration of voriconazole (A), individual effect of PPIs use on trough concentration of voriconazole (B), and combined effect of both CYP2CI9 genotype and PPIs use on trough concentration of voriconazole $(\mathbf{C})$. The dotted line indicates the maximum recommended therapeutic range of voriconazole trough concentration. NM, normal metabolizers; IM, intermediate metabolizers; PM, poor metabolizers. Score 2 = NM/PPI non-user group; score $3=$ NM/PPIs user or IM/PPIs non-user group; score 4 = IM/ PPIs user or PM/PPIs non-user group; score 4 = PM/PPIs user group. 
Table 2 Distribution of PPI Use in Patients with Different CYP2C19 Phenotype

\begin{tabular}{|l|l|l|}
\hline CYP2CI9 Genotype & $\begin{array}{l}\text { PPI Non-Users, } \\
\text { n (\%) }\end{array}$ & $\begin{array}{l}\text { PPI Users, } \\
\text { n (\%) }\end{array}$ \\
\hline Normal metabolizer, n (\%) & $19(48.7)$ & $20(51.3)$ \\
\hline $\begin{array}{l}\text { Intermediate metabolizers, } \\
\text { n (\%) }\end{array}$ & $28(53.8)$ & $24(46.2)$ \\
\hline Poor metabolizer, n (\%) & $9(39.1)$ & $14(60.9)$ \\
\hline
\end{tabular}

Abbreviation: PPI, proton pump inhibitors.

(2.19 to $9.51 \mu \mathrm{g} / \mathrm{mL}$ ) was observed in patients with total score of 2, 3, 4 and 5 respectively. The median trough concentration of voriconazole for each group was statistically significantly different from one another ( $\mathrm{p}$ between groups ranged from 0.01 to $\mathrm{p}<0.0001)$. As expected, the median trough concentration of voriconazole in PM/PPIs user (total score of 5) patients was the highest compared to other groups and the value lied above the normal recommended therapeutic range of $0.5 \mu \mathrm{g} / \mathrm{mL}$ to $5 \mu \mathrm{g} / \mathrm{mL}$ (Figure $1 \mathrm{C}$ ).

\section{Distribution of PPIs Use in Patients with Different CYP2C19 Genotype}

We observed that, out of 23 patients with PM phenotype, 14 (61\%) of them were administered with PPIs (Table 2); of which $64 \%$ of the administered PPIs in PM patients was either omeprazole or esomeprazole (Table 3). On the other hand, $24(46 \%)$ of 52 patients with IM phenotype were administered with PPIs while the proportion of NM patients administered with PPIs was 51\% (Table 3). The proportion of PM patients administered with PPIs was significantly higher than that of their counterparts PPIs non-users $(p<0.001)$.

\section{Impacts of Individual PPIs on Trough Concentration of Voriconazole}

The current study demonstrated a significant elevation of trough concentration of voriconazole in patients coadministered with either omeprazole or esomeprazole than in those administered with either lansoprazole or pantoprazole (Figure 2). The plasma concentration of voriconazole was increasing with PPIs coadministration in the order of esomeprazole $>$ omeprazole $>$ lansoprazole $>$ pantoprazole. Since PPIs use in patients exhibiting CYP2C19 PM phenotype was associated with supra-therapeutic levels of voriconazole (refer to Figure 1C), we further investigated the impact of individual PPIs on inducing supra-therapeutic levels in groups of CYP2C19 phenotype; by conducting subgroup analysis. Sub-group analysis (by excluding cases administered with either omeprazole or esomeprazole) resulted in significant decline in the median trough concentration of voriconazole to below supra-therapeutic levels in PM/PPIs users group (Figure 3); indicating that the supratherapeutic levels observed earlier were largely contributed by either omeprazole or esomeprazole.

\section{Frequency of Supra-Therapeutics Levels of Voriconazole in Patients with Different CYP2C19 Phenotype}

Supra-therapeutic levels of voriconazole (plasma concentration $>5 \mu \mathrm{g} / \mathrm{mL}$ ) was observed in all groups of CYP2C19 phenotype. However, the frequency of supra-therapeutic levels was significantly higher (47.8\%) in PM phenotype than in either NM (15.4\%) or IM (13.5\%) phenotypes, $\mathrm{p}=$ 0.002 . The number of patients with supra-therapeutic levels in PM phenotype was relatively equal to the number of patients with plasma concentration below the supra-therapeutic levels (Table 4).

\section{Determination of Factors Influencing Voriconazole Trough Concentration}

On further analysis to explore various clinical and demographic factors influencing plasma trough concentrations of voriconazole, we performed a multivariate linear regression model. Various factors including patient's age, total bilirubin, total bile acid, C-reactive protein, albumin, route of

Table 3 Distribution of PPI Type Use in Patients of Different CYP2CI9 Phenotype

\begin{tabular}{|l|l|l|l|l|}
\hline \multirow{2}{*}{ CYP2C19 Genotype } & \multicolumn{4}{|l|}{ PPI Types, $\mathbf{n}(\%)$} \\
\cline { 2 - 5 } & Omeprazole & Lansoprazole & Pantoprazole & Esomeprazole \\
\hline Normal metabolizer & $8(40)$ & $5(25)$ & $3(15)$ & $4(20)$ \\
Intermediate metabolizer & $9(37.5)$ & $6(25)$ & $1(4.2)$ & $8(33.3)$ \\
Poor metabolizer & $7(50)$ & $4(28.6)$ & $1(7.1)$ & $2(14.3)$ \\
\hline
\end{tabular}

Abbreviation: PPI, proton pump inhibitors. 


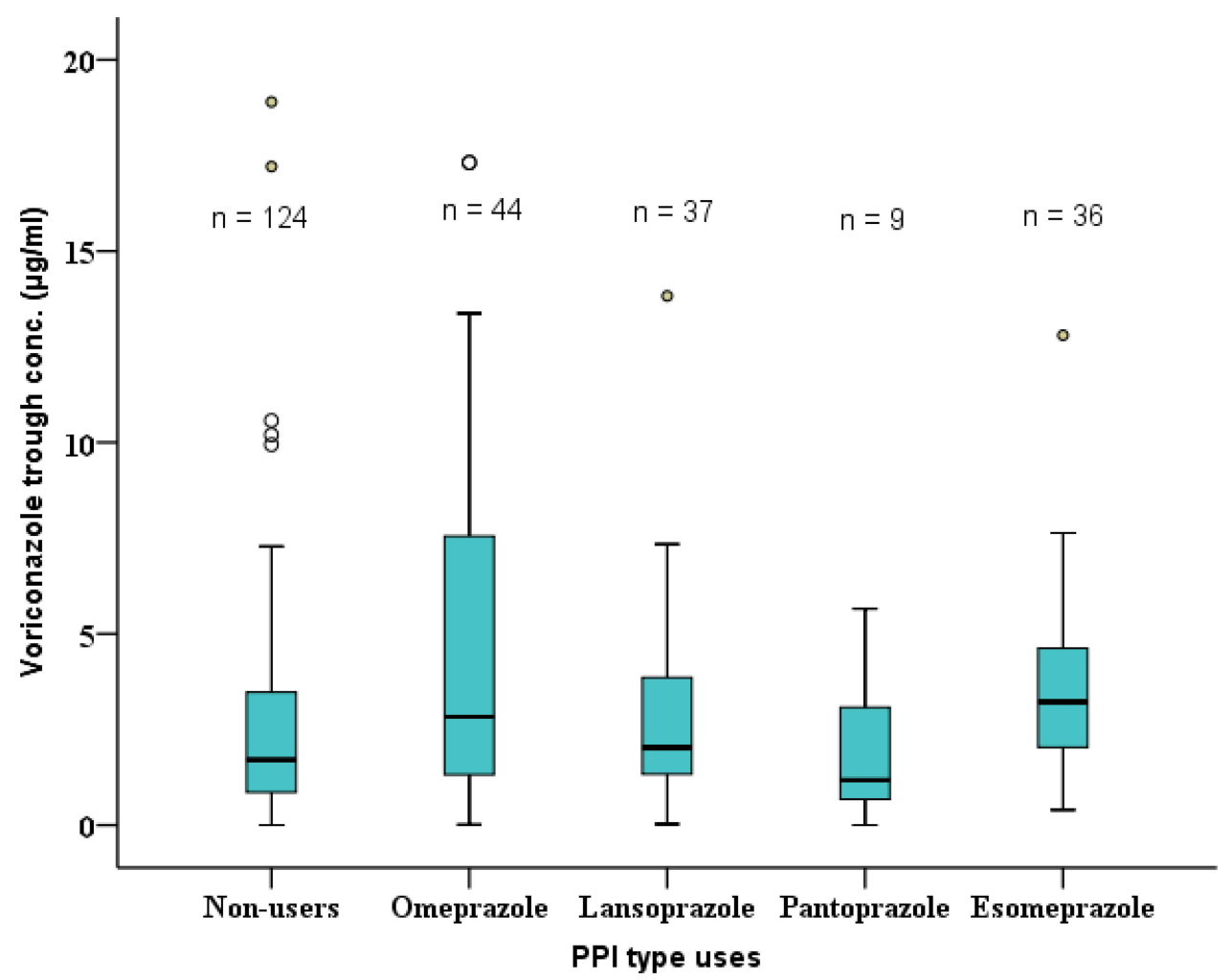

Figure 2 Box-and-whisker plot showing the Effect of individual proton pump inhibitor (PPIs) coadministration on trough concentration of voriconazole. Box (25th, median, and 75 th percentiles) and whisker (5th and 95th percentiles); the small filled and unfilled circle shows outlier cases. $P$ were $0.005 * 0.183,0.336$ and $<0.0001 *$ for omeprazole, lansoprazole, pantoprazole and esomeprazole respectively when compared with PPIs non-user group.

administration, co-morbidity, CYP2C19 phenotype, PPIs use and CYP2C19 phenotype/PPIs use combination (combined total score) were included in both univariate and multivariate linear regression model. After adjusting for confounding factors, patient's age, severe aplastic anemia, CYP2C19 PM Phenotype, PPIs uses and CYP2C19 PM phenotype/ PPIs use combination emerged as independent factors influencing trough concentration of voriconazole. Importantly, we observed that patients with CYP2C19 PM phenotype who use PPIs had approximately 5-fold higher risk of high trough concentration compared to patients exhibiting CYP2C19 NM phenotype who do not use PPIs (Table 5).

\section{Discussion}

The current study investigated the impact of the combined effect of CYP2C19 polymorphism and PPIs use on the plasma trough concentration of voriconazole. The use of individual PPIs across patients with different CYP2C19 variant alleles was also explored. CYP2C19 gene polymorphism analyzed as a single factor showed a significant impact on plasma trough concentration of voriconazole. Higher plasma concentration in PM patients than in NM individuals was noted; however, the median trough concentration ranged within the recommended therapeutic range of voriconazole in the Chinese population (ie, $0.5 \mu \mathrm{g} / \mathrm{mL}$ to $5 \mu \mathrm{g} / \mathrm{mL}) .^{22,23}$ But the addition of PPIs on top of CYP2C19 polymorphism in PM/PPIs user patients significantly elevated the median trough concentration to above the recommended therapeutic range and indicated an additive effect. Our finding revealed that patients exhibiting CYP2C19 poor metabolizer phenotype who also comedicated with PPIs were more likely to have supratherapeutic concentration of voriconazole than patients with other CYP2C19 phenotype who received or did not receive PPIs. In addition, a multivariate linear regression model demonstrated approximately 5-fold higher trough concentration of voriconazole in PM/PPI user when compared to NM/PPIs non-user patients. To the best of our knowledge, this is the first study to report the combined effect of CYP2C19 genetic polymorphism and PPIs coadministration on the trough concentration of voriconazole. The metabolism of voriconazole by CYP2C19 $9^{23,24}$ is normally reduced in patients carrying CYP2C19*2/*2, *2/*3, or $* 3 / * 3$ variant alleles. The reduced activity of the 


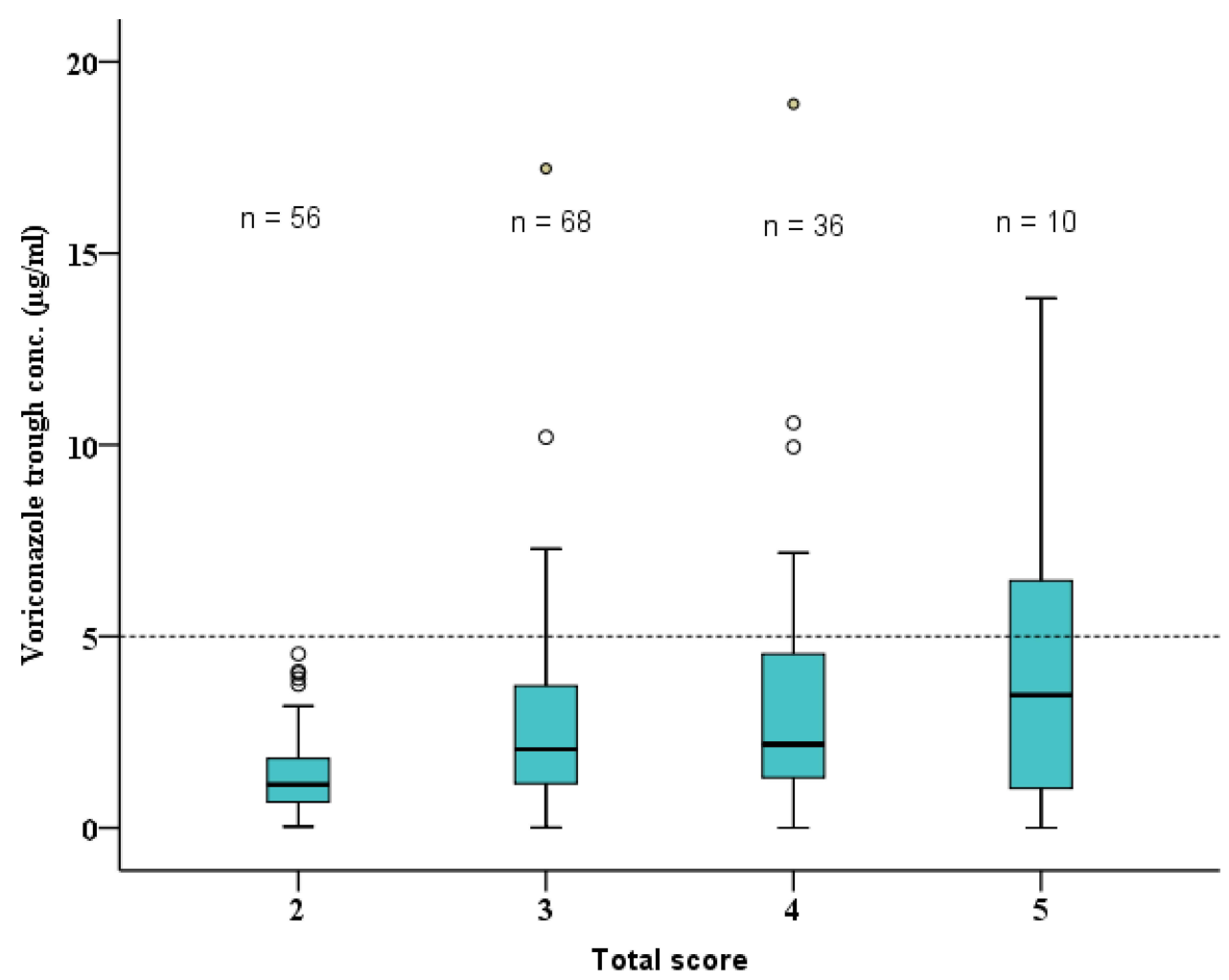

Figure 3 Box-and-whisker plot showing sub-group analysis for effect of combined effect of CYP2CI9 and PPIs use (excluding omeprazole and esomeprazole user cases) on trough concentration of voriconazole. Box (25th, median, and 75th percentiles) and whisker (5th and 95th percentiles); the small filled and unfilled circle shows outlier cases. The dotted line indicates the maximum recommended therapeutic range of voriconazole trough concentration. Score $2=$ NM/PPIs non-user group; score $3=$ NM/PPIs user or IM/PPIs non-user group; score 4 = IM/PPIs user or PM/PPIs non-user group; score 4 = PM/PPIs user group.

enzyme might be even much more when PPIs are coadministered, because PPIs confer inhibitory effect to the enzyme.

The proportion of individuals with CYP2C19 poor metabolizer phenotype has been found higher in East Asian (15 to $30 \%$ ) than in Caucasians and African Americans. ${ }^{7}$ Voriconazole and PPIs coadministration in

Table 4 Frequency of Supra-Therapeutic Levels of Voriconazole Across CYP2C19 Phenotype

\begin{tabular}{|l|c|c|}
\hline \multirow{2}{*}{$\begin{array}{l}\text { CYP2C19 } \\
\text { Phenotype }\end{array}$} & $\begin{array}{c}|c| \\
\text { Voriconazole Trough Conc. Category } \\
\text { Therapeutic Levels ( } \\
\mathbf{5 \mu g} / \mathbf{m L}), \mathbf{n}(\%)\end{array}$ & $\begin{array}{c}\text { Supra-Therapeutic } \\
\text { Levels (> 5 } \mathbf{\mu g} / \mathbf{m L}), \\
\mathbf{n}(\%)\end{array}$ \\
\hline $\begin{array}{l}\text { Normal } \\
\text { Metabolizer }\end{array}$ & $33(84.6)$ & $6(15.4)$ \\
\hline $\begin{array}{l}\text { Intermediate } \\
\text { Metabolizer }\end{array}$ & $45(86.5)$ & $7(13.5)$ \\
\hline $\begin{array}{l}\text { Poor } \\
\text { Metabolizer }\end{array}$ & $12(52.2)$ & $11(47.8)$ \\
\hline
\end{tabular}

our patients was common. About $50 \%$ of patients administered with voriconazole were also receiving PPIs for the treatment of acid-related gastric disorders, and among PPI users, $24 \%$ of them exhibited a CYP2C19 PM phenotype. Previous studies have demonstrated that, PPIs owe their clinical efficacy to their ability to inhibit $\mathrm{H}^{+} / \mathrm{K}^{+}$-adenosine triphosphatase in gastric parietal cells, resulting in suppression of gastric acid secretion, ${ }^{25}$ and the efficacy of gastric acid suppression among individual PPIs differs. For instance, in some studies, esomeprazole at the standard dose provided more effective control of gastric acid at a steady-state than standard doses of other PPIs. ${ }^{25,26}$ The variation in acid suppression efficacy of individual PPIs may influence the choice of PPIs as it can be reflected in our study $(65 \%$ received omeprazole or esomeprazole while less proportion received lansoprazole or pantoprazole).

The current study demonstrated a high trough concentration of voriconazole in omeprazole or esomeprazole users than in lansoprazole or pantoprazole users, indicating a strong inhibition of CYP2C19 enzyme by the former drugs compared to the latter drugs. Our findings concur 
Table 5 Multivariate Mixed-Effect Linear Regression Model Showing Factors Influencing Voriconazole Trough Concentration $(\mathrm{n}=250)$

\begin{tabular}{|c|c|c|c|c|}
\hline \multirow[t]{2}{*}{ Variable } & \multicolumn{2}{|l|}{ Univariate Analysis } & \multicolumn{2}{|c|}{ Multivariate Final Model } \\
\hline & $\beta$-Coefficients ( \pm SE) & $P$ value & $\beta$-Coefficients ( \pm SE) & $P$ value \\
\hline Age (yr) & $0.03 \pm 0.013$ & $0.007^{* *}$ & $0.027 \pm 0.013$ & $0.033^{*}$ \\
\hline Total bilirubin $(\mu \mathrm{mol} / \mathrm{L})$ & $(-) 0.004 \pm 0.038$ & 0.923 & & \\
\hline Total Bile acid $(\mu \mathrm{mol} / \mathrm{L})$ & $0.038 \pm 0.019$ & 0.058 & & \\
\hline C-reactive protein (mg/L) & $0.002 \pm 0.007$ & 0.749 & & \\
\hline Albumin $(g / L)$ & $(-) 0.016 \pm 0.36$ & 0.649 & & \\
\hline \multicolumn{5}{|l|}{ Co-morbidity } \\
\hline Acute Myeloid Leukemia & Reference & & & \\
\hline Acute Lymphoblastic Leukemia & $0.001 \pm 0.602$ & 0.997 & & \\
\hline Myelodysplastic Syndrome & $0.336 \pm 0.857$ & 0.696 & & \\
\hline Chronic Myeloid Leukemia & $1.216 \pm 0.975$ & 0.213 & & \\
\hline Severe Aplastic Anemia & $(-) 1.14 \pm 0.228$ & $0.0004 * * *$ & $(-) 2.305 \pm 0.584$ & $0.003 * *$ \\
\hline \multicolumn{5}{|l|}{ CYP2C19 Phenotype } \\
\hline Normal metabolizer & Reference & & & \\
\hline Intermediate metabolizer & $0.211 \pm 0.412$ & 0.609 & & \\
\hline Poor metabolizer & $2.88 \pm 0.478$ & $<0.000$ I*** & $2.344 \pm 0.725$ & $0.00 I^{* *}$ \\
\hline \multicolumn{5}{|l|}{ PPIs uses } \\
\hline Non-users & Reference & & & \\
\hline Users & $1.109 \pm 0.397$ & $0.006 * *$ & $0.959 \pm 0.478$ & $0.044^{*}$ \\
\hline \multicolumn{5}{|l|}{ Route of administration } \\
\hline Oral & Reference & & & \\
\hline Intravenous & $0.163 \pm 0.512$ & 0.75 & & \\
\hline \multicolumn{5}{|l|}{ CYP2C19/PPIs use combination } \\
\hline NM/PPI non-user & Reference & & & \\
\hline NM/PPI user or IM/PPI non-user & $0.464 \pm 0.41$ & 0.263 & & \\
\hline IM/PPI user or PM/PPI non-user & $0.635 \pm 0.45$ & 0.159 & & \\
\hline PM/PPI user & $3.5 I \pm 0.59$ & $<0.000$ I*** & $4.608 \pm 0.677$ & $<0.0001 * * *$ \\
\hline
\end{tabular}

Note: ***, and $* * *$ statistically significant at $\mathrm{p}<0.05,<0.01$ and $<0.001$ levels, respectively.

Abbreviations: PPIs, proton pump inhibitors; NM, normal metabolizer; IM, intermediate metabolizer, PM, poor metabolizer; SE, standard error.

with the reports from previous studies ${ }^{8,9,11,16}$ which reported a higher CYP2C19 inhibitory capacity by omeprazole or esomeprazole when compared to either lansoprazole, rabeprazole or pantoprazole, and the inhibitory potency of the former drugs present a clinical relevance. Contrary to the previous studies ${ }^{8,9,11,16}$ which were basically preclinical studies or model simulation, our study was basically a clinical study involved extraction of data from really patients.

The contribution of PPIs particularly omeprazole or esomeprazole in the elevation of voriconazole trough concentration to supra-therapeutic levels in PM/PPIs user patients was demonstrated. The decline of trough concentration to below supra-therapeutic levels after excluding patients administered with either omeprazole or esomeprazole, indicated that supra-therapeutic levels were largely contributed by omeprazole or esomeprazole. This finding provides clinical relevance in the choice of PPIs during coadministration with voriconazole in patients with unknown CYP2C19 genotype status. PPIs are known inhibitors of CYP2C19, however, not all PPIs inhibit CYP2C19 enzyme to the same extent; which impedes similarities in the extent of drug-drug interactions. Previous studies have demonstrated superior affinity and inhibition of CYP2C19 by esomeprazole and omeprazole than other PPIs. ${ }^{8,27}$ Therefore, the potential magnitude for a clinically relevant drug-drug interaction with voriconazole may not be generalized to all PPIs. Hence, the proper choice of PPIs may need careful consideration in the case of voriconazole coadministration in a population with a higher CYP2C19 poor metabolizer phenotype. 
It should be noted that our study had some limitations. First, CYP3A4 which also involved in metabolism of voriconazole was not genotyped, and therefore its influence on trough concentration of voriconazole was not measured. However, previous reports show that CYP3A4 plays a rather minor role, because the affinity of voriconazole to CYP3A4 is about 50-fold lower than to CYP2C19. Other limitations include lack of PPI dosing information, lack of adequate numbers for some PPI subgroups, lack of CYP2C19*17 allele genotyping, and the study was limited to Chinese population.

\section{Conclusion}

PPIs coadministration particularly omeprazole or esomeprazole in hematological patients administered with voriconazole was common. Coadministration of Proton pump inhibitors with voriconazole resulted in elevation of $\mathrm{VCZ}-\mathrm{C}_{\text {trough }}$ to supra-therapeutic levels in patients with CYP2C19 PM phenotype. Omeprazole or esomeprazole appeared to have higher contribution in the elevation of VCZ-C $\mathrm{C}_{\text {trough }}$ to supratherapeutic levels compared to lansoprazole or pantoprazole. Therefore, in health-care facilities whereby CYP2C19 genotype-based prescribing is not feasible, omeprazole or esomeprazole should be avoided in favor of lansoprazole or pantoprazole for patients administered with voriconazole.

\section{Acknowledgments}

This research did not receive any specific grant from funding agencies in the public, commercial, or not-forprofit sectors. We thank the China Scholarship Council for financial support of this study. We also thank the management of Union Hospital of Huazhong University of Science and Technology (HUST) for granting permission to conduct this study.

\section{Disclosure}

All authors declare that there are no conflicts of interest.

\section{References}

1. Lamoureux F, Duflot T, Woillard JB, et al. Impact of CYP2C19 genetic polymorphisms on voriconazole dosing and exposure in adult patients with invasive fungal infections. Int $J$ Antimicrob Agents. 2016;47(2):124-131. doi:10.1016/j.ijantimicag.2015.12.003

2. Levin MD, den Hollander JG, van der Holt B, et al. Hepatotoxicity of oral and intravenous voriconazole in relation to cytochrome P450 polymorphisms. J Antimicrob Chemother. 2007;60(5):1104-1107. doi: $10.1093 / \mathrm{jac} / \mathrm{dkm} 330$

3. Gautier-Veyret E, Fonrose X, Tonini J, et al. Variability of voriconazole plasma concentrations after allogeneic hematopoietic stem cell transplantation: impact of cytochrome $\mathrm{P} 450$ polymorphisms and comedications on initial and subsequent trough levels. Antimicrob Agents Chemother. 2015;59(4):2305-2314. doi:10.1128/AAC.04838-14
4. Veringa A, ter Avest M, Span LFR, et al. Voriconazole metabolism is influenced by severe inflammation: a prospective study. $J$ Antimicrob Chemother. 2017;72(1):261-267. doi:10.1093/jac/dkw349

5. Wang T, Zhu H, Sun J, et al. Efficacy and safety of voriconazole and CYP2C19 polymorphism for optimised dosage regimens in patients with invasive fungal infections. Int $J$ Antimicrob Agents. 2014;44 (5):436-442. doi:10.1016/j.ijantimicag.2014.07.013

6. Hyland R, Jones BC, Smith DA. Identification of the cytochrome P450 enzymes involved in the N -oxidation of voriconazole. Drug Metab Dispos. 2003;31(5):540-547. doi:10.1124/dmd.31.5.540

7. Scholz I, Oberwittler H, Riedel KD, et al. Pharmacokinetics, metabolism and bioavailability of the triazole antifungal agent voriconazole in relation to CYP2C19 genotype. Br J Clin Pharmacol. 2009;68 (6):906-915. doi:10.1111/j.1365-2125.2009.03534.x

8. Zvyaga T, Chang SY, Chen C, et al. Evaluation of six proton pump inhibitors as inhibitors of various human cytochromes P450: focus on cytochrome P450 2C19. Drug Metab Dispos. 2012;40(9):1698-1711. doi:10.1124/dmd.112.045575

9. Ogilvie BW, Yerino P, Kazmi F, et al. The proton pump inhibitor, omeprazole, but not lansoprazole or pantoprazole, is a metabolism-dependent inhibitor of CYP2C19: implications for coadministration with clopidogrel. Drug Metab Dispos. 2011;39 (11):2020-2033. doi:10.1124/dmd.111.041293

10. Yan M, Wu ZF, Tang D, et al. The impact of proton pump inhibitors on the pharmacokinetics of voriconazole in vitro and in vivo. Biomed Pharmacother. 2018;108:60-64. doi:10.1016/j.biopha.2018.08.121

11. Niece KL, Boyd NK, Akers KS. In vitro study of the variable effects of proton pump inhibitors on voriconazole. Antimicrob Agents Chemother. 2015;59(9):5548-5554. doi:10.1128/AAC.00884-15

12. Ikemura K, Hiramatsu S, Okuda M. Drug repositioning of proton pump inhibitors for enhanced efficacy and safety of cancer chemotherapy. Front Pharmacol. 2017;8:1-5. doi:10.3389/fphar.2017.00911

13. De Milito A, Fais S. Tumor acidity, chemoresistance and proton pump inhibitors. Future Oncol. 2005;1(6):779-786. doi:10.2217/ 14796694.1.6.779

14. Azzarito T, Venturi G, Cesolini A, Fais S. Lansoprazole induces sensitivity to suboptimal doses of paclitaxel in human melanoma. Cancer Lett. 2015;356(2):697-703. doi:10.1016/j.canlet.2014.10.017

15. De Milito A, Iessi E, Logozzi M, et al. Proton pump inhibitors induce apoptosis of human B-cell tumors through a caspase-independent mechanism involving reactive oxygen species. Cancer Res. 2007;67 (11):5408-5417. doi:10.1158/0008-5472.CAN-06-4095

16. Qi F, Zhu L, Li N, Ge T, Xu G, Liao S. Influence of different proton pump inhibitors on the pharmacokinetics of voriconazole. Int $J$ Antimicrob Agents. 2017;49(4):403-409. doi:10.1016/j. ijantimicag.2016.11.025

17. Hashemizadeh Z, Badiee P, Malekhoseini SA, Raeisi Shahraki H, Geramizadeh B, Montaseri H. Observational Study of Associations between Voriconazole Therapeutic Drug Monitoring, Toxicity, and Outcome in Liver Transplant Patients. Antimicrob Agents Chemother. 2017;61(12):1-10. doi:10.1128/AAC.01211-17

18. Pfizer Canada Inc. VFEND (Voriconazole) product monograph; 2016:1-58. Available from: https://www.pfizer.ca/sites/default/files/ 202102/Vfend_PM_E_243931_01-Feb-2021.pdf (accessed on October 26, 2021).

19. Wang T, Yan M, Tang D, et al. Therapeutic drug monitoring and safety of voriconazole therapy in patients with Child-Pugh class $\mathrm{B}$ and $\mathrm{C}$ cirrhosis: a multicenter study. Int $J$ Infect Dis. 2018;72:49-54. doi:10.1016/j.ijid.2018.05.009

20. Hassan A, Burhenne J, Riedel KD, et al. Modulators of very low voriconazole concentrations in routine therapeutic drug monitoring. Ther Drug Monit. 2011;33(1):86-93. doi:10.1097/FTD.0b013e31820530cd

21. Caudle KE, Dunnenberger HM, Freimuth RR, et al. Standardizing terms for clinical pharmacogenetic test results: consensus terms from the Clinical Pharmacogenetics Implementation Consortium (CPIC). Genet Med. 2017;19(2):215-223. doi:10.1038/gim.2016.87 
22. Chen K, Zhang X, Ke X, Du G, Yang K. Individualized medication of voriconazole: a practice guideline of the Division of Therapeutic Drug Monitoring, Chinese Pharmacological Society. Ther Drug Monit. 2018;40(6):663-674. doi:10.1097/FTD.0000000000000561

23. Jin H, Wang T, Falcione BA, et al. Trough concentration of voriconazole and its relationship with efficacy and safety: a systematic review and meta-analysis. $J$ Antimicrob Chemother. 2016;71 (7):1772-1785. doi:10.1093/jac/dkw045

24. Zonios D, Yamazaki H, Murayama N, et al. Voriconazole metabolism, toxicity, and the effect of cytochrome P450 2C19 genotype. J Infect Dis. 2014;209(12):1941-1948. doi:10.1093/infdis/jiu017

25. Miner P, Katz PO, Chen Y, Sostek M. Gastric acid control with esomeprazole, lansoprazole, omeprazole, pantoprazole, and rabeprazole: a five-way crossover study. Am J Gastroenterol. 2003;98 (12):2616-2620. doi:10.1016/j.amjgastroenterol.2003.09.053
26. Röhss K, Lind T, Wilder-Smith C. Esomeprazole $40 \mathrm{mg}$ provides more effective intragastric acid control than lansoprazole $30 \mathrm{mg}$, omeprazole $20 \mathrm{mg}$, pantoprazole $40 \mathrm{mg}$ and rabeprazole $20 \mathrm{mg}$ in patients with gastro-oesophageal reflux symptoms. Eur J Clin Pharmacol. 2004;60(8):531-539. doi:10.1007/s00228-004-0804-6

27. Frelinger AL, Lee RD, Mulford DJ, et al. A randomized, 2-period, crossover design study to assess the effects of dexlansoprazole, lansoprazole, esomeprazole, and omeprazole on the steady-state pharmacokinetics and pharmacodynamics of clopidogrel in healthy volunteers. J Am Coll Cardiol. 2012;59(14):1304-1311. doi:10.1016/j.jacc.2011.12.024

28. Mafuru M, Wu S, He S, Lu X, Huang J, Jiang H. The influence of proinflammatory cytokines on voriconazole trough concentration in patients with different forms of hematologic disorders. $J$ Clin Pharmacol. 2019;59(10):1340-1350. doi:10.1002/jcph.1422

\section{Publish your work in this journal}

Pharmacogenomics and Personalized Medicine is an international, peer-reviewed, open access journal characterizing the influence of genotype on pharmacology leading to the development of personalized treatment programs and individualized drug selection for improved safety, efficacy and sustainability. This journal is indexed on the American Chemical Society's Chemical Abstracts Service (CAS). The manuscript management system is completely online and includes a very quick and fair peer-review system, which is all easy to use. Visit http://www.dovepress.com/testimonials.php to read real quotes from published authors. 